\title{
Ramseyan Properties of Graphs
}

\author{
by \\ Ermelinda DeLaVina \\ delavina@math.uh.edu \\ Siemion Fajtlowicz \\ siemion@math.uh.edu \\ University of Houston, Houston, Texas 77024
}

Submitted: February 19, 1996; Accepted: August 28, 1996

\begin{abstract}
.
Every graph of chromatic number $k$ with more than $k(r-1)(b-1)$ vertices has a $b$-element independent set of vertices such that if any two of them are joined by an edge then the chromatic number stays the same or a $r$-element independent set of vertices such that joining any two of them by an edge increases the chromatic number.
\end{abstract}

\section{Introduction.}

Let $P$ be a class of graphs and let $x$ and $y$ be a pair of non-adjacent distinct vertices of $G \in P$. For every graph consider a fixed coloring of all pairs $\{x, y\}$ of nonadjacent vertices - we color $e=\{x, y\}$ blue if $G+\{e\}$ belongs to $P$, and red - otherwise. A class, i.e., a property $P$ of graphs will be called Ramseyan if large graphs from $P$ contain large monochromatic subgraphs. It is easy to see that $P$ is Ramseyan if and only if large enough graphs from $P$ have large independent sets. The necessity of this condition is obvious, and the sufficiency follows from Ramsey 
theorem applied to the red-blue coloring of 2-element subsets of a large independent set.

Given a Ramseyan class of graphs $P$ we put $P(r, b)$ to be the smallest integer $n$ such every graph from $P$ with $n$ or more elements contains a r-element red clique or a b-element blue clique. Graphs of maximum order without this property will be called $P(r, b)$-critical.

The goal of this paper is to prove the following

Theorem. If $P$ is the class of all graphs of chromatic number $k$ then $P(r, b)=$ $1+k(r-1)(b-1)$.

Our theorem was conjectured by the first author who also proved it in some cases and established all lower bounds. Examples of critical $P(r, b)$ graphs are disjoint unions of $b-1$ copies of of complete $k$-partite graphs in which every part has $r-1$ vertices. Other critical graphs can be obtained by adding extra edges to these examples but the description of all critical graphs may be quite difficult. The first author characterized all such graphs for chromatic number 2 and 3, [D].

Before proving our theorem, we shall describe an interpretation extending the usual illustration of Ramsey Theorem: a fraternity gives a get-acquainted party subject to the rule that guests may be at will separated in rooms containing only mutual strangers. If the fraternity has 3 rooms and 13 guests then either there are three guests such that if any two of them will get acquainted then the party can continue, or there are three guests such that if any two of them will get acquainted then the party must end. 
THE ELECTRONiC JOURNAL OF COMBINATORICS 3 (1996), \#R26

\section{The Proof.}

Let $B(E)$ be the set of blue edges of $G=(V, E)$ and let $B(G)$ be the graph $(V, B(E))$. The graph $R(G)$ is defined similarly with respect to the red edges.

Lemma. If $P$ is the class of all graphs of chromatic number $k$ then $R(E)$ is the intersection of all equivalence relations corresponding to $k$-colorings of $G$.

Proof: Let $\pi_{c}$ be the equivalence relation corresponding to $k$-coloring $c$ of $G$. If $\{u, v\}$ is a red edge then every $k$-coloring of $G$ assigns to $u$ and $v$ the same color, i.e. the set of edges of $R(G)$ is contained in the intersection of all equivalence relations of the form $\pi_{c}$. Conversely if $\{u, v\}$ is not red then either $u$ is adjacent to $v$ in which case every $k$-coloration of $G$ separates $u$ from $v$, or the nonedge $\{u, v\}$ is blue, in which case the chromatic number of $G+\{u, v\}$ is again $k$ and thus there is at least one $k$-coloration of $G$ which separates $u$ from $v$. Thus $R(E)$ is the intersection of all equivalence relations of the form $\pi_{c}$, where $c$ is a $k$-coloring of $G$.

Consequently $R(E)$ is an equivalence relation and we have

Corollary. $R(G)$ is a union of disjoint cliques.

Proof of the theorem: Let $G$ be a graph of chromatic number $k$ with more than $k(r-1)(b-1)$ vertices, and let $I_{j}, j=1 . . k$, be a partition of vertices of $G$ into independent sets. By the lemma every component of $R(G)$ is contained in one of $I_{j}$ 's, and by the Corollary, each $I_{j}$ is partitioned into red cliques.

Since $G$ has more than $k(r-1)(b-1)$ vertices, one of the independent sets $I_{j}$ has more than $(r-1)(b-1)$ vertices and thus it either contains a red clique with $r$ vertices or at least b disjoint red cliques. In the latter case let $S$ be a selector of the partition $I_{j}$, i.e., let $S$ meets each of the red cliques of $I_{j}$ at one vertex. $S$ has 
THE ELECTRONiC JOURnAL OF COMBINATORICs 3 (1996), \#R26

at least $b$ vertices, and since it is independent and disjoint with $R(E)$ it must form a blue clique, which proves the theorem.

\section{Problems and Conjectures}

So far we have studied very few Ramseyan properties, but in the case of trianglefree class $T$, jointly with Professor Erdos we have shown that the numbers $T(k, k)$ are at most of order $k^{3}$, and we think that similar results will hold true for $K_{n}$-free graphs. Specifically we conjecture that if $P$ is the class of $K_{n}$-free graphs then the best lower bounds for $P(r, b)$ can be obtained by taking disjoint unions of $(b-1)$ many copies of maximal (with respect to the number of edges) critical Ramsey graphs $R(n, b)$.

Some Ramsey graphs are also critical with respect to our theorem. For example the unique regular, 8-vertex graph containing no $K_{4}$ nor 3 -element independent sets is $P(3,2)$-critical in the class of all 4 -chromatic graphs.

The structure of the red graph is obvious by Corollary, but we do not know which graphs can occur as blue graphs and what are the properties of graphs in which the graph $R(G)$ is empty. There are many examples of these red-reduced graphs, and in a number of problems, it is enough to consider the factor-graph $G / R(E)$ whose vertices are equivalence classes of $R(E)$, two being adjacent if and only if they contain adjacent representatives. This operation may considerably simplify some problems and it is extensively used in [D].

The operations of taking the blue and the red graphs are partial complements

of $G$. It would be interesting to determine the identities, and equational varieties of unary algebras whose universe is class of at most countable graphs and operations are those of taking the complement, the red and the blue graphs. The motivation 
for this problem is that identities satisfied by a graph may give a considerable insight into its structure. For example, $B(G)=R R(G)$ if and only if $G$ is complete $k$-partite for some $k$.

The Dalmatian version of Graffiti made number of conjectures about operations $R$ and $B$ (derived from the chromatic number) and we included a sample of them in the first draft of this paper. Since then, most of these conjectures were settled in $[B]$ and two of them were also refuted by a referee of this paper. These conjectures and a number of newer conjectures about triangle-free graphs can be found in [W].

\section{References}

[B] Bela Bollobas and Oliver Riordan, On Some Conjectures of Graffiti, preprint 1996.

[D] Ermelinda DeLaVina, PhD dissertation, in preparation.

[R] Ronald L. Graham, Bruce L. Rothschild and Joel H. Spencer, Ramsey Theory, John Wiley and Sons, 1990.

[W] Written on the Wall, a list of conjectures of Graffiti. 